\title{
Iffy beliefs: Conditional thinking and belief change
}

\author{
CONSTANTINOS HADJichristidis \\ University of Trento, Trento, Italy \\ Simon J. HANDLey \\ University of Plymouth, Plymouth, England \\ Steven A. Sloman \\ Brown University, Providence, Rhode Island \\ Jonathan St. B. T. Evans \\ University of Plymouth, Plymouth, England \\ AND \\ David E. Over and Rosemary J. STevenson \\ Durham University, Durham, England
}

\begin{abstract}
The ability to entertain possibilities and draw inferences about them is essential to human intelligence. We examine the hypothesis that conditional if-then statements trigger a mental simulation process in which people suppose the antecedent (if statement) to be true and evaluate the consequent (then statement) in that context. On the assumption that supposing an event to be true increases belief that the event has occurred or will occur, this hypothesis is consistent with the claim that evaluating a conditional will heighten belief in its antecedent more than in its consequent. Two experiments, employing conditionals of the form If animal $A$ has property $X$, then animal $B$ will have property $X$, in which $X$ was a property that people could not readily relate to the animals, supported this claim. The effect was stronger following the evaluation of conditionals with dissimilar animal categories.
\end{abstract}

People frequently consider hypothetical possibilities (such as What if true peace is achieved in the Middle East?) and even counterfactual possibilities (such as What if true peace had been achieved in the Middle East prior to 2001?). Even children love the "what if" game. What these kinds of thoughts have in common is that they concern other possibilities; they involve drawing inferences about a possibility that is different from the one we experience. How people consider such possibilities is the subject of work on pretense (see, e.g., Nichols \& Stich, 2000), fantasy (see, e.g., Markovits, 1995), counterfactual thought (see, e.g., Byrne, 2002), causal learning and inference (Sloman, 2005), and conditional reasoning (see, e.g., Evans \& Over, 2004). Work on conditional reasoning is perhaps the most general, since it concerns how people draw inferences that are conditioned on some assumption - in other words, how we reason about if-then statements. All studies of reasoning about possibilities can be framed this way.

A recent theory of how we reason about if-then statements, the suppositional theory of conditionals (Evans
\& Over, 2004; Evans, Over, \& Handley, 2005; Handley, Evans, \& Thompson, 2006), suggests that conditionals cue a mental simulation in which people suppose the antecedent (if statement) to be true and then assess their degree of belief in the consequent (then statement). This theory is based on what philosophical logicians have called the Ramsey test (Ramsey, 1931, 1990), which bears resemblance to the simulation heuristic (Kahneman \& Tversky, 1982).

Consider, for example, the conditional

If global warming continues, then the economy of Africa will be threatened.

According to the suppositional theory, the starting point for assessing belief in this conditional is to think about a possibility in which global warming continues. One may then consider the causes and consequences of global warming (e.g., increased carbon dioxide pollution, changes in the amount of rainfall) and their effects on crops and animals. For example, increased carbon dioxide pollution might cause coral reefs to die, which in turn would threaten fisheries and income from tourism. This train of thought is

C. Hadjichristidis, k.hadjichristidis@unitn.it 
one of many that might occur in the process of evaluating a conditional of this kind, but the key point is that whatever belief one might have regarding this conditional, generating that belief depends on hypothetical thinking. Specifically, it depends on a mental simulation in which the antecedent is imagined as reality and its consequences inferred and evaluated.

One implication of this theory is that people evaluate the truth of a conditional as a function of the probability of its consequent in light of its antecedent. This claim has been supported in studies that have examined the relationship between conditional evaluations and self-generated or presented truth table distributions (Evans, Handley, \& Over, 2003; Oberauer \& Wilhelm, 2003; Over \& Evans, 2003; Over, Hadjichristidis, Evans, Handley, \& Sloman, 2007). For example, Evans et al. (2003) presented participants with conditionals about cards illustrated with colored shapes, such as If the shape is a circle then it is yellow. Participants were required to judge the probability that the conditional would be true of a card drawn at random from a pack of cards that had a given frequency distribution, such as 1 yellow circle, 4 yellow diamonds, 16 red circles, and 16 red diamonds

The resulting probability of conditional judgments was compared with corresponding objective conditional probabilities of the consequent given the antecedent (e.g., the proportion of cards with yellow circles out of all cards with circles), and a strong positive association was found. Strong positive associations have also been found in studies that compared probability of conditional judgments with corresponding judgments of conditional probability (see Hadjichristidis et al., 2001).

In the present article, we focus on a novel hypothesis, consistent with the suppositional account, which links to research concerning the cognitive effects of mere comprehension or supposition. Gilbert (1991), in support of the views of the philosopher Baruch Spinoza $(1677,1982)$, provided evidence that our belief systems are jury-rigged: We tend to accept propositions whose truth value we either cannot determine or lack sufficient resources to determine. Rejecting, or in Gilbert's terms "unaccepting," propositions is a product of a willful and effortful process that comes later, if ever. Gilbert noted that the tendency to accept propositions is also consistent with the views of several linguists (e.g., Grice, 1975) who have posited that people tend to communicate true information and act as if others do so as well (for empirical evidence, see Swann, Giuliano, \& Wegner, cited in Gilbert, 1991). Explanations for this tendency range from behavioral (people act this way because societies place heavy penalties on lying; see, e.g., Bok, cited in Gilbert, 1991) to evolutionary (our belief systems emerged out of our perceptual systems; we tend to accept what we are told in the same way that we tend to accept what we see and hear; see, e.g., Gilbert, 1991).

Regardless of what drives this effect, it seems that acceptance precedes rejection and that rejection is possible only after a willful evaluation process. In this article, we use this phenomenon to devise a test for the suppositional theory. According to this theory, conditionals of the form if $p$ then $q$ contain the linguistic instruction to suppose that the antecedent, $p$, is true and, in that context, to evaluate the consequent, $q$. To evaluate a conditional, a person needs not only to comprehend $p$ but also to confirm $p$, if only momentarily. As a result, belief in $p$ should increase. Belief in $q$ should not necessarily increase - that depends on the outcome of the evaluation process. The predicted interaction between conditional clause and belief change we refer to as the conditional belief hypothesis. No other theory of conditionals that we are aware of makes this prediction, and this hypothesis is a very strong test of the suppositional theory.

We also test a second, related hypothesis concerning the effect of the similarity between antecedent and consequent clauses on belief change. To illustrate, consider the following conditional claims concerning blank predicates - that is, predicates that people have relatively few beliefs about:

\section{If horses have sesamoid bones, then cows will have sesamoid bones. \\ If squirrels have a left aortic arch, then rhinos will have a left aortic arch.}

How might one evaluate the likelihood that each of these statements is true? The literature on category-based induction with blank predicates suggests that a major determinant of such evaluations is the similarity between the antecedent and consequent categories: The greater the similarity, the higher the judged probability (Osherson, Smith, Wilkie, Lopez, \& Shafir, 1990; Sloman, 1993, 1998). ${ }^{1}$ We offer a parallel claim for the evaluation of conditionals that relate propositions involving blank predicates. Participants will always suppose the antecedent, $p$, to be true, and this will increase their belief in it. Belief in the consequent, $q$, will depend, in part, on the perceived degree of similarity between the antecedent and consequent categories. When similarity is high, as in Conditional 2, the act of evaluating a conditional will also increase belief in the consequent. When similarity is low, as in Conditional 3, evaluating a conditional will not affect belief in the consequent or may even decrease it. Consequently, we expect the conditional belief effect (i.e, a greater increase of belief in $p$ than in $q$ ) to interact with similarity: Dissimilar antecedent-consequent categories should result in a larger effect. We refer to this prediction as the similarity hypothesis.

We tested these hypotheses in two experiments by using a posttest-only control group design. Participants in the treatment condition of each experiment were asked first to judge the probability of conditionals (treatment), then the probability of their antecedent and consequent statements (posttest). Control participants were asked to judge the probability of antecedent and consequent statements only (posttest). We predicted that treatment would increase posttest judgments of probability for the antecedents but not necessarily for the consequents, in accord with the conditional belief hypothesis. We also varied the similarity between antecedent and consequent categories. We predicted that the conditional belief effect would be 
more pronounced for dissimilar categories, in accord with the similarity hypothesis.

\section{EXPERIMENT 1 Blank Predicates}

\begin{abstract}
Method
Participants. Eighty-nine University of Plymouth students volunteered to participate.

Materials. We selected conditionals whose antecedent and consequent statements participants were unlikely to have beliefs about. Prior evaluation of conditionals such as If antelopes have hearts (or three eyes), then elephants will have hearts (or three eyes) seems unlikely to produce belief change because people know that their constituents are true or false and will judge accordingly in the posttest. Counterfactuals are also unlikely to produce belief change, because people usually know that their antecedents are false. For this reason, we used indicative conditionals with a single believable statement and an uncertain blank predicate as the antecedent and conclusion, respectively, as in Conditionals 2 and 3. Following other studies that used blank predicates, we employed a mix of real biological properties (e.g., aortic arch, Vitamin K, choroid membrane) and made-up properties (e.g., stenozoidal cells, neurotransmitter Dihedron). For a full list of the conditionals used, see Appendix A. This type of conditional was selected for two additional reasons. First, its symmetry (both $p$ and $q$ have the form Animal $A$ has property $X$ ) aided experimental control. In Experiment 1, the only difference between $p$ and $q$ was the animal mentioned. In Experiment 2, there was no difference at all, since the position of statements serving as $p$ or $q$ was counterbalanced - that is, each statement served as its own control. Second, this type of conditional allowed us to draw on the extensive literature of related categorical arguments (see Osherson et al., 1990), such as Horses have sesamoid bones; therefore, cows will have sesamoid bones, to develop hypotheses (e.g., the similarity
\end{abstract} hypothesis) and construct materials.

Procedure. The participants in the treatment condition first rated the probability of 16 conditionals (treatment), then rated the probability of 16 unrelated statements (a filler task taking about 5 min to complete), and finally rated the probability of the antecedent and consequent statements of each of the 16 conditionals (posttest). The participants in the control group skipped the treatment phase. The participants were assigned to experimental groups at random and were tested in small groups. Participants recorded their responses in the booklets in which the tasks were presented. The first page of each booklet stated that the purpose of the study was to learn more about people's beliefs in statements, thanked the participants for taking part, and informed them that their responses would be treated confidentially. The following pages contained the items of the various tasks, with each task preceded by an instruction page. The instruction pages for the treatment and posttest tasks were similar. Participants were informed that they would be presented with a series of statements and that their task was to describe how likely to be true they found each of these statements on a scale of $0-10$, with 0 being not at all likely and 10 being very likely. Participants were given a practice item, similar in each case to the test items. Half of the conditionals in the treatment task contained similar pairs of mammals (e.g., horsescows, dolphins-seals; see Conditional 2) and half, dissimilar pairs (e.g., gorillas-deer, raccoons-lions; see Conditional 3). An example of a posttest item is, "How likely do you think it is that horses have stenozoidal cells?" The assignment of category pairs to similarity conditions was determined by a pilot study. The order of items within each task was randomized separately for each participant. For the posttest task, an additional constraint was that no two sentences from the same conditional appeared in direct succession.

\section{Results and Discussion}

Mean likelihood ratings are shown in Table 1. Our hypotheses concerned belief increase, measured as the mean likelihood increase in a treatment condition versus the increase in its respective control (treatment minus control). Consistent with our conditional belief hypothesis, there was greater belief increase in the antecedent, $p$, than in the consequent, $q(M=+.41$ vs. $M=+.18)$. Consistent with our similarity hypothesis, the gap in belief increase was more pronounced in the low- than the high-similarity condition $(M=+.32$ vs. $M=+.14)$.

We performed two 2 (condition: treatment vs. control) $\times 2$ (position: $p$ vs. $q) \times 2$ (similarity: high vs. low) ANOVAs, one across participants $\left(F_{1}\right)$ and one across items $\left(F_{2}\right)$. Consistent with the conditional belief hypothesis, belief increase was significantly higher in $p$ than in $q$, as evidenced by a significant condition $\times$ position interaction $\left[F_{1}(1,85)=4.67, p=.03, \eta_{\mathrm{p}}^{2}=.05 ; F_{2}(1,14)=4.66, p=\right.$ $\left..049, \eta_{\mathrm{p}}^{2}=.25\right]$. Specifically, belief increase was significant in $p\left[F_{1}(1,85)=6.36, p=.014, \eta_{\mathrm{p}}^{2}=.07 ; F_{2}(1,15)=8.56\right.$, $\left.p=.01, \eta_{\mathrm{p}}^{2}=.36\right]$ but not in $q\left[F_{1}(1,85)<1 ; F_{2}(1,15)=\right.$ $2.53, p=.13]$, as shown by analyses pitting treatment versus control scores. Turning to the similarity hypothesis, this effect was more pronounced in the low-similarity condition, as predicted, but not significantly so, as evidenced by the absence of a three-way interaction $(F \mathrm{~s}<1)$. The only other significant effect was a condition $\times$ similarity interaction $\left[F_{1}(1,85)=4.82, p=.031, \eta_{\mathrm{p}}^{2}=.05 ; F_{2}(1,14)=\right.$ $\left.3.48, \mathrm{p}=.08, \eta_{\mathrm{p}}^{2}=.20\right]$. In part, this effect seems to be the result of a decrease in belief in $q$ following treatment in the low-similarity condition. We return to this finding when we discuss the results of Experiment 2.

Experiment 1 examined whether prior evaluation of conditionals increased belief in their antecedents more than in their consequents (the conditional belief hypothesis). It did. It also examined whether this effect was more pronounced in the low- versus the high-similarity condition (similarity hypothesis). The data suggest such a difference, but it was not statistically significant.

\section{EXPERIMENT 2 Nonexplainable Predicates}

The main purpose of Experiment 2 was to gather further evidence for the conditional belief hypothesis and to extend it to a different type of nonexplainable predicate

Table 1

Mean Likelihood Ratings With Standard Deviations in Antecedent and Consequent $\times$ Similarity Condition for Experiment 1

\begin{tabular}{|c|c|c|c|c|}
\hline \multirow[b]{2}{*}{ Condition } & \multicolumn{2}{|c|}{ Antecedent $(p)$} & \multicolumn{2}{|c|}{ Consequent $(q)$} \\
\hline & $M$ & $S D$ & $M$ & $S D$ \\
\hline \multicolumn{5}{|l|}{ High Similarity } \\
\hline Treatment & 5.60 & 1.15 & 5.45 & 1.20 \\
\hline Control & 5.04 & 0.86 & 5.03 & 0.69 \\
\hline Belief increase (BI) & \multirow{2}{*}{\multicolumn{4}{|c|}{+.14}} \\
\hline $\mathrm{BI}(p)-\mathrm{BI}(q)$ & & & & \\
\hline \multicolumn{5}{|l|}{ Low Similarity } \\
\hline Treatment & 5.31 & 0.81 & 5.14 & 0.99 \\
\hline Control & 5.06 & 0.91 & 5.21 & 0.81 \\
\hline & +.25 & & -.07 & \\
\hline $\mathrm{BI}(p)-\mathrm{BI}(q)$ & \multicolumn{4}{|c|}{+.32} \\
\hline
\end{tabular}


(Sloman \& Wisniewski, 1992) - that is, predicates that people are familiar with but find hard to explain in relation to the categories used. For example, people know what "never travel in the direction of the sun" means, but they do not know, and cannot even explain or infer, whether this is true of salmons or lions. A second purpose was to seek evidence for the similarity hypothesis. To that end, we increased the similarity gap between high- and low-similarity category pairs by using low-similarity pairs (e.g., hippos-tunas, antelopes-dolphins) that appeared to us to be more dissimilar than those used in Experiment 1 (e.g., elephants-beavers, raccoons-lions). In Experiment 2 , high-similarity pairs were from the same superordinate (e.g., mammal-mammal, fish-fish), whereas several of the low-similarity pairs were from different superordinates (e.g., mammal-fish). A third purpose was to provide additional control for the belief change comparison between $p$ and $q$. In Experiment 1, the categories used for $p$ and $q$ statements were not counterbalanced, and this could conceivably have contributed to the observed differences in belief increase between $p$ and $q$. In Experiment 2 , the categories were counterbalanced. For instance, about half of the participants in the treatment condition received If horses like Mozart, then cows will like Mozart, whereas the remaining half received If cows like Mozart, then horses will like Mozart.

\section{Method}

Participants. A total of 128 University of Leeds students volunteered to participate.

Design, Materials, and Procedure. The design, materials, and procedure were similar to those used in Experiment 1, with the exceptions that we used nonexplainable predicates, varied similarity more widely (see above), counterbalanced the categories that appeared in antecedent and consequent positions, and did not include a filler task. For a full list of the conditionals used in Experiment 2, see Appendix B.

\section{Results and Discussion}

The results are summarized in Table 2. Consistent with our conditional belief hypothesis, there was a higher belief increase in $p$ than in $q(M=+28$ vs. $M=-.10)$. Consistent with our similarity hypothesis, this effect was more pronounced in the low- versus the high-similarity condition $(M=+.71$ vs. $M=+.05)$.

This time, both hypotheses were supported. The conditional belief hypothesis predicts greater belief increase in $p$ than in $q: \mathrm{P}_{\text {treatment }}(p)-\mathrm{P}_{\text {control }}(p)>\mathrm{P}_{\text {treatment }}(q)-$ $\mathrm{P}_{\text {control }}(q)$. In Experiment 2, this prediction reduced to $\mathrm{P}_{\text {treatment }}(p)>\mathrm{P}_{\text {treatment }}(q)$, because $\mathrm{P}_{\text {control }}(p)=\mathrm{P}_{\text {control }}(q)$. To examine the conditional belief hypothesis, we performed two 2 (position: $p$ vs. $q$ ) $\times 2$ (similarity: high vs. low) ANOVAs, one across participants $\left(F_{1}\right)$ and one across items $\left(F_{2}\right)$. As expected, $\mathrm{P}_{\text {treatment }}(p)>\mathrm{P}_{\text {treatment }}(q): M=$ 4.84 vs. $M=4.46$. This difference was statistically significant $\left[F_{I}(1,121)=3.58, p=.06\right.$ (significant if a one-tailed $t$ test is adopted), $\eta_{\mathrm{p}}^{2}=.03 ; F_{2}(1,30)=8.03, p=.008$, $\left.\eta_{\mathrm{p}}^{2}=.21\right]$. To examine the similarity hypothesis, we tested whether there was a significant interaction in the abovementioned ANOVA. There was $\left[F_{I}(1,121)=6.84, p=\right.$ $\left..032, \eta_{\mathrm{p}}^{2}=.08 ; F_{2}(1,30)=7.11, p=.012, \eta_{\mathrm{p}}^{2}=.19\right]$.
The above analyses show more belief change in $p$ than in $q$, and they show that this change was greater in the low-similarity condition. However, they do not show whether belief after treatment in each condition increased or decreased. For this, we need to compare treatment judgments with corresponding control judgments. To examine this, we ran two ANOVAs, one for $p$ and one for $q$ ratings. As in Experiment $1, \mathrm{P}_{\text {treatment }}(p)>\mathrm{P}_{\text {control }}(p)$ $\left[F_{1}(1,191)=3.18, p=.076\right.$ (significant if a one-tailed $t$ test is adopted), $\eta_{\mathrm{p}}^{2}=.02 ; F_{2}(1,30)=6.38, p=.017$, $\left.\eta_{\mathrm{p}}^{2}=.18\right]$ and $\mathrm{P}_{\text {treatment }}(q) \approx \mathrm{P}_{\text {control }}(q)\left(F_{\mathrm{s}}<1\right)$. Also as in Experiment 1, evaluating a conditional with dissimilar categories decreased belief in its consequent statement $(M=$ $-.25)$, though this effect was marginal $\left[F_{1}(1,192)=2.12\right.$, $\left.p=.15 ; F_{2}(1,15)=2.97, p=.11\right]$. This finding provides some support for our claim that dissimilar categories cue participants to entertain possibilities in which the consequent does not possess the property not- $q$ by focusing participants' attention on $p$-and-not- $q$ cases.

Comparing belief increase across experiments, more belief increase occurred in Experiment 1 (this was true in three out of four position $\mathrm{x}$ similarity cells). This might be because the predicates used in Experiment 1 were more credible (control credibility scores were about .5 points higher in Experiment 1 than were the corresponding scores in Experiment 2). Presumably, entertaining a possibility will increase belief only if the possibility is credible. Belief might actually decrease if entertaining a possibility is hard (Sherman, Cialdini, Schwartzman, \& Reynolds, 1985) or if doing so makes people think of reasons that the possibility might be false. This would explain the negative effect of supposing $q$ in the low-similarity condition. The only case in which there was more belief increase in Experiment 2 than in Experiment 1 was in the low-similarity antecedent judgments $(M=+.46$ vs. $M=$ +.25 ). This might have been noise, or, as an anonymous reviewer suggested, it might have been because when similarity is relatively high, people think mainly of $p$-and- $q$ cases. When similarity is sufficiently low, as in the lowsimilarity condition of Experiment 2, people think about

Table 2

Mean Likelihood Ratings* With Standard Deviations in Antecedent and Consequent $\times$ Similarity Condition for Experiment 2

\begin{tabular}{|c|c|c|c|c|}
\hline \multirow[b]{2}{*}{ Condition } & \multicolumn{2}{|c|}{ Antecedent $(p)$} & \multicolumn{2}{|c|}{ Consequent $(q)$} \\
\hline & $M$ & $S D$ & $M$ & $S D$ \\
\hline \multicolumn{5}{|l|}{ High Similarity } \\
\hline Treatment & 4.68 & 1.31 & 4.63 & 1.28 \\
\hline Control $^{* *}$ & 4.59 & 1.23 & 4.59 & 1.23 \\
\hline Belief increase (BI) & +.09 & & +.04 & \\
\hline $\mathrm{BI}(p)-\mathrm{BI}(q)$ & \multicolumn{4}{|c|}{+.05} \\
\hline \multicolumn{5}{|l|}{ Low Similarity } \\
\hline Treatment & 5.00 & 1.12 & 4.29 & 1.27 \\
\hline Control & 4.54 & 1.05 & 5.21 & 1.05 \\
\hline BI & +.46 & & -.25 & \\
\hline $\mathrm{BI}(p)-\mathrm{BI}(q)$ & \multicolumn{4}{|c|}{+.71} \\
\hline
\end{tabular}

${ }^{*}$ Not at all likely, 0 ; very likely, $10 .{ }^{* *}$ Because the position of statements serving as $p$ or $q$ was counterbalanced, antecedents and consequents in each similarity condition had the same control. 
both $p$-and- $q$ and $p$-and-not- $q$ cases. Entertaining both of these cases involves more (or longer) supposition of $p$, hence a larger increase in the belief in $p$.

\section{GENERAL DISCUSSION}

It has long been recognized that mental simulation and entertaining possibilities play an important role in mental life. According to the suppositional account of conditionals, the connective if has the function of inviting us to engage in exactly this sort of thinking. In two experiments, we examined whether prior evaluations of conditionals heightened belief in their antecedents more than in their consequents (the conditional belief hypothesis), a prediction that was motivated by the suppositional account of conditionals and that relates to one of its key assumptions: People suppose that $p$ is true and evaluate $q$ in that context. Our data suggest that people do make such suppositions: Conditional evaluation, like other processes involving hypothetical thinking, such as imagination, leads to belief change. In our experiments, we also examined whether this effect was more pronounced in the low-similarity condition (the similarity hypothesis). It was, but it reached significance only when we varied similarity widely in Experiment 2.

A further finding was that belief in the consequent decreased after participants evaluated a conditional with dissimilar antecedent-consequent categories, especially in Experiment 2, in which low-similarity category pairs were more dissimilar than those of Experiment 1. This finding is consistent with our claim that dissimilar category pairs lead participants to think more about $p$-and-not- $q$ cases than do similar category pairs, leading to increased belief in $p$ but decreased belief in $q$.

Our results, in line with the suppositional account, suggest that if acts as a linguistic trigger for people to engage in hypothetical thought, leading them to perform a mental simulation of the antecedent. It is perhaps surprising that people's beliefs changed at all, given that the properties that we used were either essentially devoid of assessable meaning (Experiment 1) or meaningful but unrelated to knowledge concerning the category member (Experiment 2). However, other research has also shown that simulating possibilities can lead people to believe quite unusual things, such as that bizarre imagined actions (e.g., kissing a magnifying glass) have actually occurred (Thomas \& Loftus, 2002).

The suppositional theory proposes a modified version of the simulation heuristic (Kahneman \& Tversky, 1982). According to the simulation heuristic, people judge the probability of antecedent, $p$, and consequent, $q$, statements by relying on the ease with which they can mentally construct or imagine the events described in $p$ and $q$. According to the suppositional account, people evaluate a conditional by supposing $p$ to be true and then estimating whether $q$ or not- $q$ is more likely to follow. The simulation heuristic holds that when people evaluate conditional probabilities, their judgments correspond to how easily $p$ and $q$ can be imagined; it is assumed that people do not consider not- $q$. But people do consider not- $q$ (see, e.g., Evans et al., 2003; Hadjichristidis et al., 2001; Over et al., 2007). The current finding that evaluating a conditional increases belief in $p$ more than in $q$ provides further reason to believe that not- $q$ is more likely to be considered than not- $p$.

Our findings cannot be explained by two accounts that have been proposed to explain why vivid imagination of an event can increase belief in that event. These accounts are relevant because vivid imagination could have mediated conditional evaluation (at least in Experiment 2, in which conditionals involved events that could be imagined). One account, which we term the familiarity account, attributes such imagining effects to familiarity (Garry \& Polaschek, 2000). Imagining events makes them more familiar, and it is claimed that people attribute this increased familiarity to the actual occurrence of the event. In the experiments presented here, a simple familiarity account would predict belief increase in both $p$ and $q$, since both were explicitly presented in the conditional evaluation task. However, belief increase was greater in $p$ than in $q$; in fact, belief in $q$ generally decreased following evaluation of conditionals with dissimilar antecedent-consequent categories. Another account, the imagination-reality confusion account, holds that imagining $p$ to be true makes participants lose track of the hypothetical status of their representations, leading them to believe that the antecedent is a true description of the world rather than a temporary supposition (see Koehler, 1991). This account predicts that participants in the treatment condition would not simply increase their beliefs in $p$ but would actually consider $p$ to be certain. This is not what we found.

Another issue is whether our findings are consistent with alternative theories of the meaning of conditionals, such as the mental model theory (MMT; Johnson-Laird \& Byrne, 2002). According to the MMT, people construct mental models representing true possibilities and estimate the sum of the probabilities of the models in which the event occurs (Johnson-Laird, Legrenzi, Girotto, Legrenzi, \& Caverni, 1999). The suggested core mental models for the ordinary indicative conditional if $p$ then $q$ are: $p, q$; not- $p, q$; not- $p$, not- $q$.

The MMT allows for virtually any combination of these models. MMT cannot explain why prior evaluation of a conditional raises belief in $p$ but not in $q$, because no single model (or combination of models) favors the $p$ over the $q$ possibility. If anything, the MMT should predict the opposite, because more core models contain the not- $p$-and- $q$ possibilities. Consider, for example, the claim that people think only about the $p$-and- $q$ possibility, which Johnson-Laird and Byrne (2002) have made. If this claim were true, then we would expect belief change to be equal for both the antecedent and consequent propositions. The data clearly indicate otherwise.

Recent work on conditionals has focused on the role they play in modifying listeners' beliefs (see, e.g., Thompson, Evans, \& Handley, 2005). For example, the conditional

\section{If the railway line is closed then taxpayers will save millions of dollars in maintenance costs}

has been shown to invite listeners to derive a complex argument such as "damage to the highway $q$ is undesirable and should be avoided, $q$ will occur if the railway 
line close $p$ is undertaken. If one wants to avoid $q$, then one should not take action $p$, therefore action $p$ should be avoided" (example from Thompson et al., 2005, p. 242). The present experiments imply that there is a more direct route to persuasion. Merely asking people to entertain a conditional (especially when they have few prior beliefs in its constituent statements) can increase belief in its antecedent. In the context of the present experiments, this effect seems innocuous: People increased their beliefs that animals had certain properties. But think about the consequences in situations like murder trials. Asking the jury to entertain conditionals such as If the accused is guilty . . . could tilt the jury's opinion in favor of finding the accused guilty. In such situations, iffy beliefs can lead to iffy decisions with potentially devastating consequences.

\section{AUTHOR NOTE}

This research was supported by ESRC Grant R000239074, "Belief revision and uncertain reasoning," and by NSF Award SES-0518147. We thank Klaus Oberauer and three anonymous reviewers for helpful comments on earlier versions of this article. Correspondence concerning this article should be addressed to C. Hadjichristidis, Department of Cognitive Sciences and Education, University of Trento, Polo di Rovereto, Via M. del Ben 5b, Rovereto (TN) 38068 Italy (e-mail: k.hadjichristidis@unitn.it).

\section{REFERENCES}

Byrne, R. M. J. (2002). Mental models and counterfactual thinking. Trends in Cognitive Sciences, 6, 405-445.

Evans, J. St. B. T., Handley, S. H., \& Over, D. E. (2003). Conditionals and conditional probability. Journal of Experimental Psychology: Learning, Memory, \& Cognition, 29, 321-355.

Evans, J. ST. B. T., \& Over, D. E. (2004). If. Oxford: Oxford University Press.

Evans, J. St. B. T., Over, D. E., \& Handley, S. J. (2005). Suppositions, extensionality, and conditionals: A critique of the mental model theory of Johnson-Laird and Byrne (2002). Psychological Review, 112, 1040-1052.

Garry, M., \& PolascheK, D. L. L. (2000). Imagination and memory. Current Directions in Psychological Science, 9, 6-10.

Gilbert, D. T. (1991). How mental systems believe. American Psychologist, 46, 107-119.

GrICE, H. P. (1975). Logic and conversation. In P. Cole \& J. L. Morgan (Eds.), Syntax and semantics, Vol. 3: Speech acts (pp. 41-58). New York: Seminar Press.

Hadichristidis, C., Stevenson, R. J., Over, D. E., Sloman, S. A., Evans, J. St. B. T., \& Feeney, A. (2001). On the evaluation of if $p$ then $q$ conditionals. In Proceedings of the Twenty-Third Annual Conference of the Cognitive Science Society (pp. 381-386). Mahwah, NJ: Erlbaum.

Handley, S. J., Evans, J. St. B. T., \& Thompson, V. A. (2006). The negation of conditionals: A litmus test for the suppositional conditional? Journal of Experimental Psychology: Learning, Memory, \& Cognition, 32, 559-569.

HeIt, E. (1998). A Bayesian analysis of some forms of inductive reasoning. In M. Oaksford \& N. Chater (Eds.), Rational models of cognition (pp. 248-274). Oxford: Oxford University Press.
Johnson-Laird, P. N., \& Byrne, R. M. J. (2002). Conditionals: A theory of meaning, pragmatics, and inference. Psychological Review, 109, 646-678

Johnson-Laird, P. N., Legrenzi, P., Girotto, V., Legrenzi, M. S., \& CAVERNI, J.-P. (1999). Naive probability: A mental model theory of extensional reasoning. Psychological Review, 106, 62-88.

Kahneman, D., \& TVersky, A. (1982). The simulation heuristic. In A. Kahneman, P. Slovic, \& A. Tversky (Eds.), Judgment under uncertainty: Heuristics and biases (pp. 201-210). Cambridge: Cambridge University Press.

Koenler, D. J. (1991). Explanation, imagination, and confidence in judgment. Psychological Bulletin, 110, 499-519.

Markovits, H. (1995). Conditional reasoning with false premises: Fantasy and information retrieval. British Journal of Developmental Psychology, 13, 1-11.

Nichols, S., \& STich, S. (2000). A cognitive theory of pretense. Cognition, 74, 115-147.

Oberauer, K., \& Wilhelm, O. (2003). The meaning(s) of conditionals: Conditional probabilities, mental models, and personal utilities. Journal of Experimental Psychology: Learning, Memory, \& Cognition, 29, 680-693.

Osherson, D., Smith, E. E., Wilkie, O., Lopez, A., \& Shafir, E. (1990). Category-based induction. Psychological Review, 97, 185200.

Over, D. E., \& Evans, J. St. B. T. (2003). The probability of conditionals: The psychological evidence. Mind \& Language, 18, 340-358.

Over, D. E., Hadichristidis, C., Evans, J. ST. B. T., Handley, S. J., \& Sloman, S. A. (2007). The probability of causal conditionals. Cognitive Psychology, 54, 62-97.

Ramsey, F. P. (1990). General propositions and causality. In D. H. Mellor (Ed.), Philosophical papers (pp. 145-163). Cambridge: Cambridge University Press. (Original work published 1931)

Sherman, S. J., Cialdini, R. B., Schwartzman, D. F., \& Reynolds, K. D. (1985). Imagining can heighten or lower the perceived likelihood of contracting a disease: The mediating effect of ease of imagery. Personality \& Social Psychology Bulletin, 11, 118-127.

SLoman, S. A. (1993). Feature-based induction. Cognitive Psychology, 25, 231-280.

Sloman, S. A. (1998). Categorical inference is not a tree: The myth of inheritance hierarchies. Cognitive Psychology, 35, 1-33.

Sloman, S. A. (2005). Causal models: How people think about the world and its alternatives. New York: Oxford University Press.

Sloman, S. A., \& WisniewsKi, E. J. (1992). Extending the domain of feature-based models of property induction. In Proceedings of the Fourteenth Annual Conference of the Cognitive Science Society (pp. 355-359). Mahwah, NJ: Erlbaum.

SpINOZA, B. (1982). The ethics and selected letters. (S. Feldman, Ed., \& S. Shirley, Trans.). Indianapolis, IN: Hackett. (Original work published 1677)

Thomas, A. K., \& Loftus, E. F. (2002). Creating bizarre false memories through imagination. Memory \& Cognition, 30, 423-431.

Thompson, V. A., Evans, J. St. B. T., \& Handley, S. J. (2005). Persuading and dissuading by conditional argument. Journal of Memory \& Language, 53, 238-257.

\section{NOTE}

1. Different theorists have assumed different mechanisms for computing similarity, including featural overlap (e.g., Sloman, 1993) and relative frequency of $p$-and- $q$ over $p$-and-not- $q$ cases in terms of known properties of the categories (e.g., Heit, 1998). Our account for the conditional could be construed in terms of any of these accounts. 


\section{Conditionals Used to Construct the Items of Experiment 1}

\section{High Similarity}

If horses have stenozoidal cells, then cows will have stenozoidal cells.

If mice use Dihedron as a neurotransmitter, then squirrels will use Dihedron as a neurotransmitter.

If dolphins require titanium for normal development, then seals will require titanium for normal development.

If rhinos require vitamin $\mathrm{K}$ for their livers to function, then elephants will require vitamin $\mathrm{K}$ for their livers to function.

If chimps require magnesium for reproduction, then gorillas will require magnesium for reproduction.

If lions have a lower body temperature in infancy than in maturity, then tigers will have a lower body temperature in infancy than in maturity.

If deer secrete uric acid crystals, then antelopes will secrete uric acid crystals.

If beavers have an ulnar artery, then raccoons will have an ulnar artery.

\section{Low Similarity}

If seals require oxydilic acid for good digestion, then horses will require oxydilic acid for good digestion.

If cows have a choroid membrane, then mice will have a choroid membrane.

If elephants require biotin for hemoglobin synthesis, then beavers will require biotin for hemoglobin synthesis.

If squirrels have a left aortic arch, then rhinos will have a left aortic arch.

If gorillas have a liver with two chambers, then deer will have a liver with two chambers.

If tigers have a higher potassium concentration in their blood than humans do, then chimps will have a higher potassium concentration in their blood than humans do.

If antelopes have sesamoid bones, then dolphins will have sesamoid bones.

If raccoons use streptokinase as an enzyme, then lions will use streptokinase as an enzyme.

\section{APPENDIX B}

\section{Conditionals Used to Construct the Items of Experiment 2}

Two lists of conditionals were used, the one below and one in which the position of statements serving as $p$ or $q$ was reversed.

\section{High Similarity}

If horses like Mozart, then cows will like Mozart.

If dolphins sleep with one eye open, then seals will sleep with one eye open.

If mice are either "left-handed" or "right-handed," then squirrels will be either "left-handed" or "righthanded."

If rhinos give birth under water, then hippos will give birth under water.

If alligators cannot stick their tongues out, then crocodiles cannot stick their tongues out.

If tunas' dorsal fins act as fleshy bait to attract smaller fish as prey, then salmons' dorsal fins will act as fleshy bait to attract smaller fish as prey.

If lions dream while sleeping, then tigers will dream while sleeping.

If deer warn members of their group by hopping up with four stiff legs, then antelopes will warn members of their group by hopping up with four stiff legs. 


\section{APPENDIX B (Continued)}

\section{Low Similarity}

If seals, two weeks after birth, can move as fast as adults, then horses, two weeks after birth, can move as fast as adults.

If cows use their ears to help dissipate excess body heat, then mice will use their ears to help dissipate excess body heat.

If hippos change to a slightly darker color during winter to maintain body temperature, then tunas will change to a slightly darker color during winter to maintain body temperature.

If squirrels have very poor memories, then rhinos will have very poor memories.

If crocodiles have a common exit point for feces and urine, then deer will have a common exit point for feces and urine.

If tigers have extraordinary hearing, then alligators will have extraordinary hearing.

If antelopes suffer from frequent migraines, then dolphins will suffer from frequent migraines.

If salmons never travel directly in the direction of the sun, then lions will never travel directly in the direction of the sun.

(Manuscript received October 17, 2006;

revision accepted for publication February 16, 2007.) 